\title{
What Matters in Professional Drone Pilots' Practice? An Interview Study to Understand the Complexity of Their Work and Inform Human-Drone Interaction Research
}

\author{
Sara Ljungblad \\ Interaction Design, Computer Science \\ and Engineering (CSE), University of \\ Gothenburg and Chalmers University \\ of Technology \\ Gothenburg, Sweden \\ sara.ljungblad@chalmers.se \\ Mafalda Gamboa \\ Interaction Design, CSE, Chalmers \\ University of Technology \\ Gothenburg, Sweden \\ mafalda.gamboa@chalmers.se
}

\author{
Yemao Man \\ Interaction Design, CSE, University of \\ Gothenburg and Chalmers University \\ of Technology \\ Gothenburg, Sweden \\ yemao.man@chalmers.se
}
Mohammad Obaid
Interaction Design, CSE, Chalmers
University of Technology
Gothenburg, Sweden
mobaid@chalmers.se

\author{
Mehmet Aydın Baytaş \\ t2i lab, Interaction Design, CSE, \\ Chalmers University of Technology \\ Gothenburg, Sweden \\ baytas@chalmers.se
}

\author{
Morten Fjeld \\ t2i lab, Interaction Design, CSE, \\ Chalmers University of Technology \\ Gothenburg, Sweden and University \\ of Bergen \\ Bergen, Norway \\ fjeld@chalmers.se
}

\begin{abstract}
Human-drone interaction is a growing topic of interest within HCI research. Researchers propose many innovative concepts for drone applications, but much of this research does not incorporate knowledge on existing applications already adopted by professionals. This limits the validity of said research. To address this limitation, we present our findings from an in-depth interview study with 10 professional drone pilots. Our participants were armed with significant experience and qualifications - pertinent to both drone operations and a set of applications covering diverse industries. Our findings have resulted in design recommendations that should inform both ends and means of human-drone interaction research. These include, but are not limited to: safety-related protocols, insights from domain-specific use cases, and relevant practices outside of handson flight.
\end{abstract}

\section{CCS CONCEPTS}

- Human-centered computing $\rightarrow$ Ubiquitous and mobile devices; Interaction devices.

\section{KEYWORDS}

Drones, quadcopters, piloting, interview study, professionals, pilots

\section{ACM Reference Format:}

Sara Ljungblad, Yemao Man, Mehmet Aydın Baytaş, Mafalda Gamboa, Mohammad Obaid, and Morten Fjeld. 2021. What Matters in Professional Drone Pilots' Practice? An Interview Study to Understand the Complexity

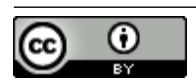

This work is licensed under a Creative Commons Attribution International 4.0 License.

CHI '21, May 8-13, 2021, Yokohama, Japan

(c) 2021 Copyright held by the owner/author(s).

ACM ISBN 978-1-4503-8096-6/21/05.

https://doi.org/10.1145/3411764.3445737 of Their Work and Inform Human-Drone Interaction Research. In CHI Conference on Human Factors in Computing Systems (CHI '21), May 813, 2021, Yokohama, Japan. ACM, New York, NY, USA, 16 pages. https: //doi.org/10.1145/3411764.3445737

\section{INTRODUCTION}

Human-Drone Interaction ${ }^{1}$ (HDI) is a growing topic of interest in $\mathrm{HCI}$ research $[4,5,8,42]$. The emerging use of drones is already affecting society and different types of practices. Drones are now an integrated part of some work practices (see Figure 1), services and leisure activities. Being knowledgeable about the nature of such everyday technological practices is important for the $\mathrm{CHI}$ community, in order to produce relevant research. So far, drones have been investigated in domain areas such as sports and wellbeing $[27,28,35]$, construction and structure inspection [17, 41], rescue operations $[1,19,39]$, accessibility [3], smart homes and companionship [20,30], logistics [11]. However, an overall account of professional perspectives of drone practices is missing.

A significant body of the HDI research within $\mathrm{HCI}$ is interventional: researchers introduce new artifacts to address particular design challenges or opportunities. These conceptual and visionary investigations are worthwhile contributions, but they share a common limitation: success and scalability for the many proposed use cases is dependent on technological advances or design innovations that are extrinsic to the investigation at hand. Thus, for example, the majority of empirical studies on autonomous drones are performed via Wizard of $\mathrm{Oz}$ (WoZ) procedures, with piloted drones (i.e. the implementation of the proposed autonomous functionality is beyond current means); and some with no physical drones at all. From recent HDI literature reviews [4, 42], it appears that only a small number of empirical HDI research publications describe the

\footnotetext{
${ }^{1}$ We use the term "drone" to refer to flying robots. While the quadcopter form factor is mostly common in current drone applications, we do not exclude other forms of drones, e.g. blimp and winged designs.
} 


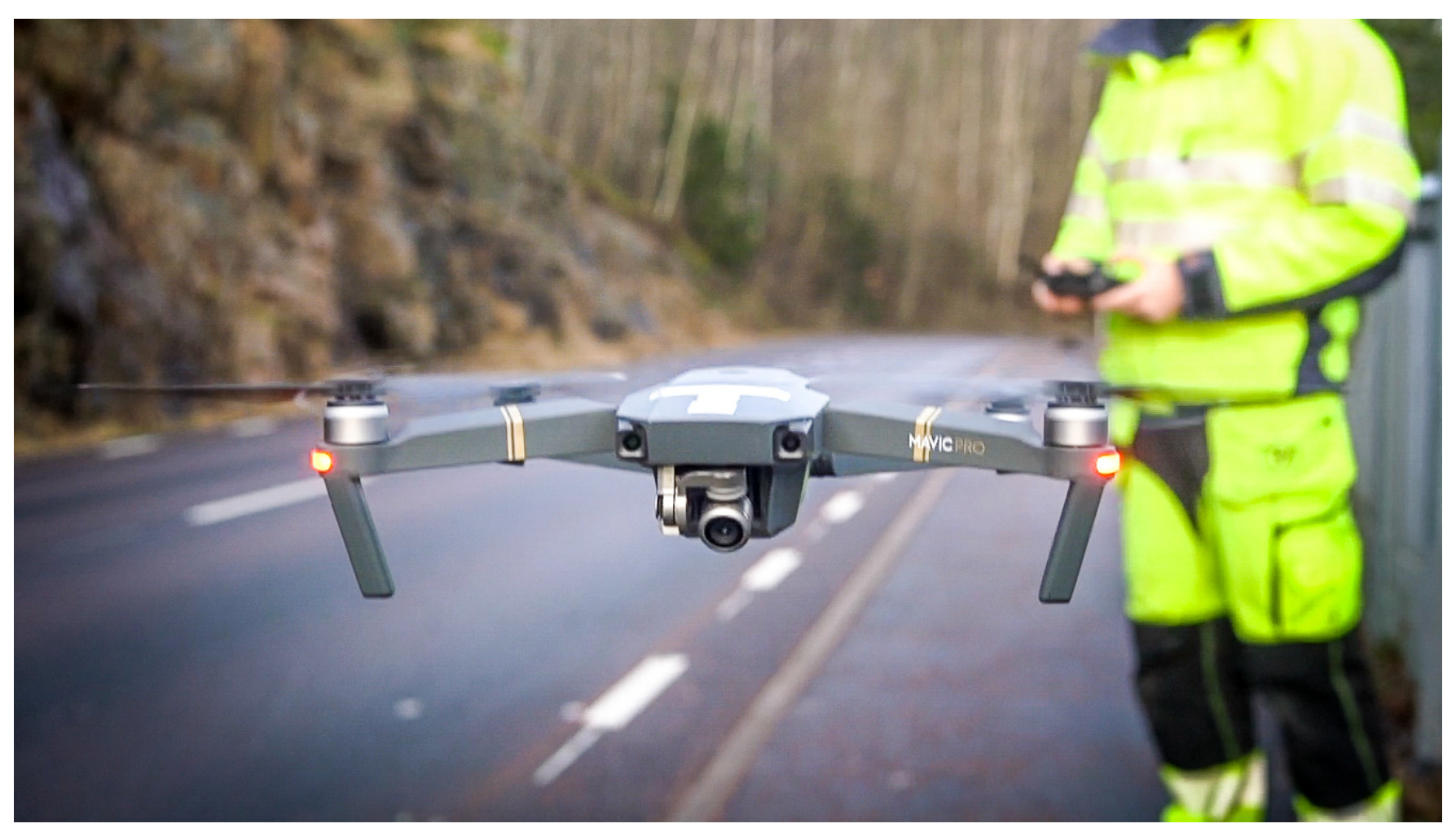

Figure 1: Drones are becoming integrated in work practices.

details of piloting procedures or how safety measures and liability protection are implemented in studies with co-located drones.

The trajectory of current HDI research suggests that the research community may benefit from a perspective covering real-world, practical, and professional considerations relevant for drones. Although they are not as ubiquitous in everyday life as artifacts like smartphones or cars, pilot-operated drones are used in a wide range of commercial and hobbyist applications, utilized by many professionals and enthusiasts on a daily basis. These use cases include, but are not limited to, photography, racing, construction, police work, forestry, infrastructure inspection, emergency response, and agriculture. The experiences and wisdom of professional drone users, the perspective of those most knowledgeable and practiced in the actual piloting of drones, should yield insights that contribute to HDI research. In the absence of this perspective, HDI research runs the risk of disputes with respect to the ecological validity of research results, as well as the validity of many guiding assumptions and scenarios. As a result, for example, the impacts of interventional concepts on bystanders and other stakeholders might be misguided. As this paper will show, the input from professional practitioners may also corroborate and inform considerations relevant to novel HDI concepts.

Towards this end, we present an interview study with 10 pilots who habitually fly drones for professional purposes. The goal with the study was to learn about how these professionals describe their practices and priorities. As we articulate below, these pilots have experience and expertise in using drones in real-world context, interacting with diverse stakeholders and bystanders, responding to tactical risks, taking account of regulations, and more. They have received systematic training on drones and aviation. They have a direct and rich perception of what is needed, accomplished, and lacking in the current HDI designs. To the best of our knowledge, this work has not been carried out before, in particular by characterizing drone pilots' state of practice and relating it to the state of the art in HDI research; with the purpose to identify opportunities, constraints, and best practices that may inform HDI research. Thus, the novel contribution of this paper is two-fold:

(1) We present a rare account of the experiences and expertise of professional drone pilots in a variety of industries. We describe their practices, education, and priorities.

(2) We extract insights to inform HDI research and design, from the aforementioned account. We reveal opportunities and directions for future HDI research and give design recommendations. We observe that the state of practice and the state of HDI research often diverge, and we offer a resource for HDI researchers who may wish to align with the state of practice.

\section{RELATED WORK}

Related literature that addresses HDI considerations in practice is sparse and, in this section, we shed-light on the few related studies that investigated drones in real world contexts. We specify how our work builds on the related work. 
Restas [33] reports on a tactical analysis for how drones might be useful in five disaster scenarios, while Brennan et al. [7] discuss HDI use cases for remote collaboration and design concerns for "wilderness search and rescue" operations. These works articulate the value of drones in tactical operations in different use scenarios, but do not report on pilots' experiences in handling such situations in their practice.

Moreover, Wang et al. [46] have interviewed a "diversified set" of 16 participants who received a brief presentation on drone functions and capabilities, as well as privacy scenarios. Participants were concerned about drones peeking and stalking (physical privacy), and recording and sharing information (information privacy). Moreover, the participants would like to see that regulation would make it illegal to fly drones without integrity adaptations. Participants also suggested that drones used by the police should have specific color, to signify purposes or ownership in order to be trusted. Our study exemplifies how professional drone pilots are much less concerned about privacy, but follow regulations and care for safety. We also show that different types of signage to signify the professional practice and involvement of stakeholders (such as the police) was essential.

On the other hand, Yao et al. [48] conducted an interview study with 12 drone controllers to investigate their perception and practices in relation to privacy concerns. Their study revealed several findings in the context of privacy, however, they did not investigate general practices of drone pilots outside the scope of privacy. Moreover, the range of pilot experiences, education, or professions that the interviewees represent is also unclear. It appears that these pilots were mostly hobbyists, while in our work, we focus on understanding professional pilots that have experience in controlling drones in the field of their expertise. Similarly, in another study, Yao et al. [49] conducted an online study with 169 pilots and 717 bystanders to gauge an understanding on how privacy mechanisms are perceived. In contrast, we chose to adopt an interview based approach, similar to [48], to conduct in-depth and semi-structured interviews, providing us with rich data on how experienced professionals articulate their own priorities.

Hildebrand [16] reports on 8 interviews, ethnographic fieldwork and cyber-ethnographies on hobbyist drone pilots (photographers and videograpers), aiming to "situate" their practices "alongside people, things, and data in physical and virtual spheres". The study reveals that some hobby pilots pay attention to follow regulations, and make on site considerations for safety and respect of others (such as flying early to avoid other people). Hildebrand also describes how pilots engage in atmospheric relations (e.g. weather and satellite), geographic relations (e.g. power lines) and mobile relations (e.g. classifications of drones), which are affected by regulatory relations. This is something that resonates with our study, clarifying how professional pilots carefully plan and conduct missions with a variety of considerations, such as safety and regulations.

Khan and Neustaedter [21,22] have done an exploratory study to collect the opinions of laypeople and firefighters to reveal potentials of how drones might assist the work of firefighters. The firefighters were positive about using drones in situations involving hazardous material, or to detect chemicals with sensors, representing risky situations. Using a drone to capture a scene was also considered beneficial. Similarly, our study reveals how the value of drones typically concerns adding value to an existing practice, such as providing a safer alternative to dangerous tasks, or providing an overview of a work situation.

More recently, Tezza et al. [43] have surveyed 515 first-personview (FPV) pilots to uncover preferences and to "give human-drone interaction researchers an understanding of the FPV community and how pilots interact with the drones" [43]. They concentrate on FPV flying as a hobby, while we focus on professionally motivated applications.

Finally, from the related work and our observations, we have identified a research gap and an opportunity to contribute to the HDI community by investigating drone pilots' practices in professional contexts. As we present our results below, we highlight relations and comparisons with related work, and give design recommendations for HDI research.

\section{STUDY DESCRIPTION}

We conducted an interview study to characterize the practices and priorities of professional drone pilots-the state of practice in professional HDI. Moreover, we were interested in comparing this state of practice and professional future outlook to the state of the art in HDI research. Thus we aimed to identify opportunities, constraints, and best practices that may inform both ends and means in HDI research and design.

All interviews were conducted during the summer of 2020, via video-conferencing. Before settling on interviews as our research method, we also considered ethnographic observation and online surveys. We preferred interviews to get more qualitative data and ask follow up questions. Furthermore, surveys that revealed various quantifiable features of the civilian drone landscape exist already (e.g. [13, 43, 49]), while qualitative aspects - e.g. how pilots describe their own practices and experiences, and the meanings they make from them [36] - called for further investigation. The other possibility, in situ ethnographic observations, was desirable, but was not feasible as the goal was to have a diverse set of pilots that operated in different contexts, also including a variety of countries.

We iteratively developed a set of 23 questions for the interviews. The process included: discussions among the research team; discussions with drone pilots with whom we were acquainted; comparisons to previous HDI research, as well as other HCI, design, and human factors research where interviews were utilized (e.g. $[12,14,15,23,34,44,47]$; and consulting methodological resources (e.g. [10]). We aimed to achieve a mix of very basic questions which requested descriptions of practices, priorities, anecdotes; questions about pilots' future outlook; and questions which followed from HDI research, aiming to uncover how the state of practice compares to research trends. Some of our questions addressed a combination of these aims. The final question set is given on Table 2, covering five primary categories: pilot demographics and experience, flight contexts and use cases, drone behavior and functions, pilot practices, and business.

\subsection{Participants and Demographics}

Participant recruitment comprised reaching out on opportunistic sampling, looking for alumni of drone piloting education programs, 
Table 1: Information on our participants.

\begin{tabular}{llllll}
\hline & Location & Age & Gender & Role & Organization \\
\hline P1 & China & 29 & Male & Tech Lead & Aerial Data Analytics Company \\
P2 & Sweden & 22 & Male & Photographer, Filmmaker & Self-employed \\
P3 & Sweden & 54 & Female & Drone Ops Consultant for Forestry & Self-employed \\
P4 & California, USA & 34 & Male & Photographer, Filmmaker, Data Analyst & Self-employed \\
P5 & Sweden & 54 & Male & Police Inspector & Police \\
P6 & California, USA & 39 & Male & Drone Cinematographer, Teacher & Drone Manufacturer and Services Provider \\
P7 & China & 47 & Male & CEO & Drone Services Provider \\
P8 & Norway & 47 & Male & Operations Manager & Energy Engineering \\
P9 & Australia & 53 & Male & Drone Ops Consultant & Self-employed \\
P10 & Sweden & 40 & Male & Rock inspector, Geologist & Engineering, design and advisory firm \\
\hline
\end{tabular}

trawling our professional networks, and posting on online communities. Snowball sampling was also utilized, as some participants recommended other participants. We observed the maximal variation principle [31], looking for individuals from different work contexts (conditions) and from different countries (location), to see if there were shared patterns across the cases. We corresponded with and screened 26 candidate participants for our interview study, interviewed 11 people, and analyzed 10 interviews. All of the interviewees in our final set of 10 participants considered themselves to be professional drone pilots, and all 10 had at least one year of first-hand, hands-on experience habitually (e.g. at least around once per week) piloting drones for professional purposes. They were considered as expert users, and our small sample size was intentional, as we prioritized "thick data" and "depth" over breadth in the study [10]. Overall, the number of participants needed for a qualitative study depends on when saturation is reached, which is affected by the research question, the empirical material, and data analysis [37]. Furthermore, previous studies of expert users have reached an acceptable result from about 5-10 participants e.g. ([32], [40]), which supported the set up with 10 expert users.

The ages of our participants ranged from 22 to 54 . They were located and operating professionally in 5 different countries: Australia, China, Norway, Sweden, and the USA. Thus, a wide range of legal and business environments are represented in their professional experiences. Table 1 and Table 3 consolidate this information.

Though a conclusive survey on the demographics of drone pilots worldwide is beyond our scope, we observe some patterns in demographics. We hoped to recruit a set of participants that represented a balanced mix of genders. However, our cohort of 10 drone pilots included 9 people who identified as male, and only one who identified as female. We also note that among 26 candidates whom we corresponded with, in our observation, only 4 were female. This pattern agrees with related work [43, 48], suggesting that the gender skewness might be representative of the larger population. Moreover, 4 out of 10 participants declared themselves as "self-employed." Though we may not speculate about the larger population based on our small sample, this proportion of self-employed professionals is drastically larger compared to 2019 OECD data for self-employment rates ${ }^{2}$ in Australia (9.7\%), Norway (6.5\%), Sweden (9.8\%) and the USA (6.1\%).

One interviewee was excluded from the final analysis as their level of experience as a pilot was found to not satisfy the recruitment criteria as a professional drone pilot (i.e. having at least one year of habitual professional flying experience).

\subsection{Data collection and Analysis}

Semi-structured interviews were conducted by three of the authors, over one-on-one video conferencing between the interviewee and the author. We followed the same protocol with each participant, going through all questions on Table 2 in the given order, but also posing follow-ups and revisiting questions as necessary, based on the information revealed to us. Interviews were recorded and usually transcribed automatically and then corrected by the author who conducted the interview. Three interviews (held in Swedish) were instead transcribed with a transcription service, and than automatically translated and corrected by the author who conducted the interview. Notes were taken during the interview to support the transcription, and clarifying annotations were made on the transcripts when necessary.

The interviews typically lasted about 1 hour (ranged from 43 minutes to 1 hour and 20 minutes). Each participant signed an informed consent, and received gift a voucher from an online bookstore worth approximately 40 USD.

Two of the authors (henceforth: "we") conducted an inductive thematic analysis [6] using MaxQDA 2020 [25, 26]. We familiarized with the material by listening to the recordings and reading the transcripts. Line-by-line coding of the first transcript was done collaboratively, to generate and discuss initial codes without any explicit protocol. This was an open discussion, where lines in the interview data were coded and collated into potential themes. This

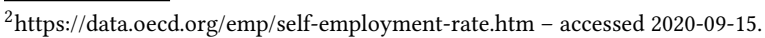


Table 2: Interview questions.

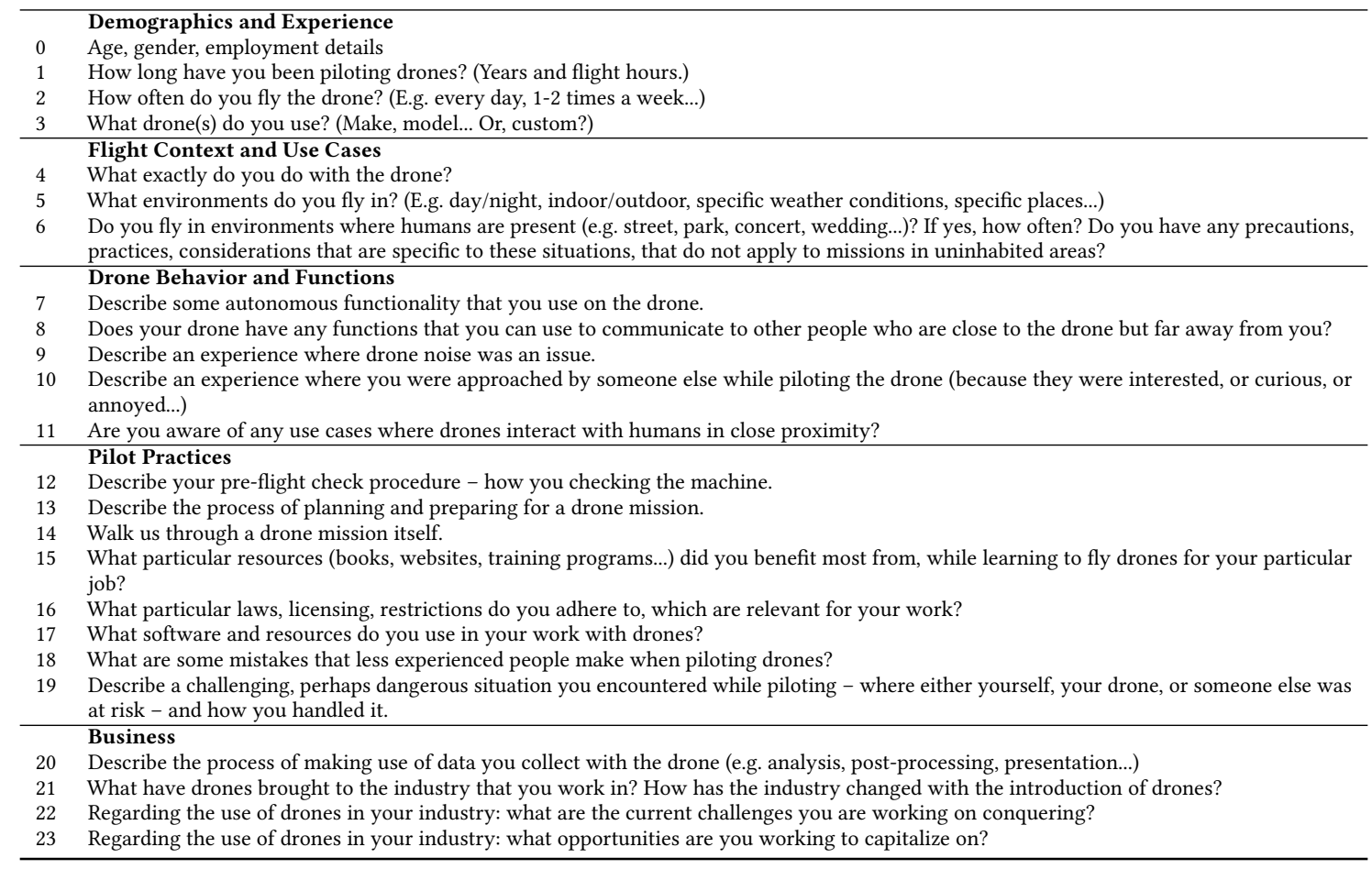

set the basis for the rest of the analysis, with deliberate efforts to build an initial code structure while still allowing for addition of new codes and restructuring the hierarchy of codes.

Afterwards, we (two of the authors) took turns to code transcripts individually. The coding researcher also entered annotations on coding considerations (i.e. why a particular line was coded in a particular way) and reflections (e.g. common and specific findings, potential themes). After coding each transcript, the coding researcher shared the set of codes with the second researcher. All codes were refined through discussions centering on the most recent analysis, and all previously analyzed transcripts were reviewed to add any new codes. Thus, previous codes were continuously cross-checked with additions. The annotations served to keep track of the analysis, accelerating each iteration. After aligning our views on all transcripts we started to code another transcript. This iterative process continued until the entire data set had been coded and analyzed.

A synthesis process followed the coding. We reviewed the codes by reading the related excerpts, and reflected on patterns, themes and differences. We also used the MaxQDA "code relations browser" to reveal the "thematic map" of the analysis - a visualisation of relationships between codes which identifies their co-occurrences [26].

\section{RESULTS}

This section presents our findings from interviews with professional drone pilots, reported with two ends in mind: First, to contribute a description of the state of practice; second, to compare this state of practice to the state of the art in HDI research, highlighting opportunities, constraints, and best practices to inform HDI research.

Our findings are organized in six subsections below. We (1) clarify the business purposes and value proposals relevant to professional drones; (2-4) describe pilots' pre-flight, flight, and post-flight workflows; (5) report on the experiences of our pilots, how they learn their craft, and how they use drones outside work; and (6) share their future outlook and expectations. For concision and clarity, implications for HDI research are given together with the findings.

\subsection{Purpose and Value of Professional Drone Applications}

Our interviews illuminate the character of and considerations for how drones create value in multiple industries. HDI researchers who wish to investigate commercial applications may follow these accounts.

From a technology perspective, in professional applications, drones are primarily imaging devices. The purpose of virtually all professional drone missions is data capture: still photos, videos, or 3D models. Thus, the ways in which drones are valuable for professional use co-evolves with other technical fields, e.g. computer vision and graphics.

None of our pilots brought up any own usage where drones perform as actuators rather than sensors. Conversely, a wealth of HDI research proposes drone movements [18, 27], drone-mounted lights, projection, or displays [9, 24, 38]; and robotic components $[2,30]$ where drones can be understood as actuators. In this way, 
HDI research and the state of industrial practices revealed in this paper seem to diverge.

From a business perspective, drone missions are often industryspecific. Drones typically facilitate or add value to an existing process, but for P3 this was still perceived as a revolution for the industry.

4.1.1 Value to Industries: Cost, Safety, Effectiveness, and Convenience. A prominent mode of how drones create value is by drastically impacting the cost or effectiveness of an existing business process. Examples include:

- Radical cost reduction (incl. time cost), e.g. in cinematography and police operations, replacing helicopters.

- Increasing safety, e.g. in inspection of hazardous environments.

- Increasing effectiveness, e.g in surveying, providing better precision and accuracy over ground-based methods.

- Decreasing intrusiveness, e.g. in inspection of wildlife in forests and oceans.

Even if the majority of these are improvements over existing concerns, these can still can be a great value creation in business For the pilot in forestry (P3), drones were expressed to enable revolution in the forestry industry.

An example where safety is the foremost value dimension is power line inspections; where historically, people had to climb high voltage towers. Often, safety and speed are improved in tandem, as in P3's forestry work: forest terrain can be studied in person over many hours, while being at risk of snake or insect bites, in a wet environment. Using a drone, P3 visits five sites in one working day, rather than just one, and feels safe while doing it. P10, a geologist who inspects quarries and roadside slopes, gives a similar example: "I would say that we use drones to go where we don't want to risk humans. Like if it's a risk of rocks falling, to look at those places, to see if there is a larger risk of rockfall. So it can get quite close and you can see. We will not send a human there and camera with a lot of zoom is not enough, so the drone is perfect for those. And sometimes there is rockfall. Which can take down the drone."

P10's engineering use cases also highlight the use of drones as imaging devices. One task that he takes up is inspecting roadside slopes, calculating probabilities and magnitudes of rockfall. They build 3D models and terrain maps, which inform reinforcement work. Similarly, P1 calculates earthwork at construction sites via photogrammetry - a task historically done manually over weeks or months. Drones images also improve the accuracy and reliability of these calculations, and allow access to challenging areas, e.g. dense vegetation. A reliable 3D model, produced with the drone, also saves many stakeholders from traveling to the site.

Different value propositions (e.g. time, cost, safety, new imaging perspectives) can combine into new workflows in a single use case. For example, in structural inspections, scaffolding may need to be constructed and later taken down. P8 obviates this work at oil refineries: "You can use drones to go to inspect areas that is normally not available for personnel when the production is going on. If you're going to send people in, you have to shut down the platform... So you can use drones to go to inspect areas that is normally not available for personnel when the production is going. And it can really decrease the use of scaffolding. Use of scaffolding is very expensive."
The results from drone missions may also be used to minimize hazards for workers, as P8 notes: "Let's say there is a large steel structure, and there is a fan on the structure that needs to be inspected, and we need to look for the integrity of all the bolts and nuts and surface conditions, regarding corrosion, cracking in the weld. Sometimes the client wants us to look for dropped objects, or potential dropped objects, [...] a wrench or a screwdriver has been forgotten in the infrastructure that can possibly fall down."

Truly novel value creation centers on being able to reach a previously inaccessible environment with a versatile imaging device, as $\mathrm{P} 9$ articulates "We have a person who's been looking at detecting old land mines from heat signatures so rescue scenarios, not putting people into these things. So the whole range of things where drones are coming in and people are becoming a back step. A lot of the time for safety reasons." And though the physical nature of aerial imaging might be novel, it often connects to existing business concerns:

In forestry, there are a lot of contractors who work, there are fellers, and there are machine operators, and someone buys timber and it has to be transported out of the forest, and so on. Then it can also be partly for the landowner to document that: "I am not happy with this job. There are folds here that have become clogged. There are logs here."

Then the landowner may take pictures with drones and send the contractor. And even the opposite, a contractor can say: "Now we have done what we can." Many forest owners are certified and have a set of rules to follow, then they can show: "When we left this forest area, we followed everything according to the certification. Here is the documentation." So, photo and film from drones, which shows clearly. Then they are covered. [P3]

Some applications are linked closely to peripheral technologies, e.g. computer graphics, and machine learning. P6 works with real estate, where they were working on combining 3D exterior drone maps with 3D interior scans of commercial real estate. This way, stakeholders would be able to take "virtual tours" where both exteriors and interiors are connected and appear realistic.

A design recommendation for HDI research: Carefully consider existing values, such as imaging, in industry to develop functionalities and applications of drones. The corollary from our findings is that there are niche opportunities for HDI research and applications within existing business concerns, across industries and problem domains. Current HDI research concentrates on hobby applications, assistive technologies, and tactical scenarios like firefighting or search and rescue. The focus is often using drones as actuators. Conversely, in commercial drone applications across traditional industries like construction, forestry, and infrastructure; significant value is created with drones as imaging devices.

4.1.2 Value to Individual Pilots: Extending Industry Expertise. An argument repeated frequently was that most value created by pilots comes from application-specific knowledge, rather than piloting skill. The value of drones as a tool serving existing industry concerns cuts across disciplines: real-estate, police, rock inspection, forestry, mining, film-making, photography and more. P3 described how forestry offices throughout Sweden are supposed to have at 
least one drone. She took up flying drones after her education in forest science, which also covered digitisation in the forest industry. Even P6, a drone cinematographer who prides himself on his advanced piloting skills, corroborates:

In the beginning, in the early days of the commercial drone industry, people believed that if you are a pilot, that you have all this inherent value. And then (...) companies started figure out - these things are actually pretty easy to fly: "I'm going to teach my guy, who knows exactly what I want to do with this data. I'll teach him, get him certified." (...) I tell people, it's not really about that you're flying. It's really taking this ability to fly commercially, and adding it to what you already do. That's what's going to bring value to you. My line of work is really in the cinematography world. So a lot of it has to do with video. I've been a Director of Photography over 15 years, and I've been doing that for a long time.

$\mathrm{P} 4$, though he is primarily a fine art photographer, made the same argument:

Pilots hope to generate a revenue or build a business around drones are forgetting that it's not about drones. It's about an industry and its problems. And if drones solves the problem, then they will be adopted. Drones have clear positive results for companies in terms of return on investment, time savings, cost cutting, safety of the employees..."

Vis-à-vis the observation that high-value drone pilots have industry-specific expertise, piloting skills also seem to transfer across industries. In addition to her work in forestry, P3 had work as a journalist specializing in drone missions, documenting fairs and events. Similarly, P9 had experience of drones in multiple industries, such as mining, film-making and photography, and P4, P6 and P7 had experience of teaching and photography.

A design recommendation for HDI research: Consider that professional pilots are foremost experts of a specific business and its values - piloting is second. Firstly, professional drone pilots are experts of a specific business, and secondly, they also fly drones in that business. The findings suggest there are fertile opportunities for application-specific HDI research and development, and also for investigating universal piloting tools and capabilities.

Typically, drone technology is operated in a very specific context, with a specific purpose, and drones are only used to add value to an existing business or operation.

\subsection{Workflow: Planning and Preparing}

In this section we describe the workflow of pilot practices. In this context, a large proportion of pilots' efforts are spent on planning and preparing. This is interesting, as most HDI research addresses tactical flight rather than strategic planning [4, 42]. The workflow of a typical drone mission, based on our participants' accounts, is illustrated in Figure 2.
4.2.1 Off-site Planning. Pilots invest significant time in planning missions. The vast majority of this work is desk research, done offsite. The details and ordering of work items vary between people and industries; but we were able to compile a typical planning workflow, given below.

(1) Initial engagement with client. Planning begins upon being contacted by a client, where they discuss the purpose and needs of the operation, to be on the same page, as P4 describes: "So I put this in a couple-page document and send it to the clients, ask them if anything is missing, so that when I'm on the spot, on the day for when I'm shooting, there's no missing information. So giving them a basically a full flight plan of what I'm going to do, how long it's going to take, the insurance that I have, the angles that I plan to cover, any waivers... Depending on the industry that they are working in, the tasks are inherently different." At this stage, the pilot has an idea of purpose, location, and specific spots of interest. Most missions are planned to be conducted outside, at daytime.

(2) Location specifics. Pilots use various software, maps, and websites to get information about the site. They assess feasibility and risk considerations. For example, are there any restricted areas nearby, such as airports or prisons? Are there power lines or other strong magnetic influences nearby? Is the terrain flat or mountainous? Are there obstructions, natural or artificial? ("And then I search manually as well... Obstacles, masts, bird nests, trees and so, nearby." [P5]) Pilots may be concerned about the presence of the bystanders. It might be required to block the area, set up certain signage, or use some other means of communication to inform people - to ensure the safety of bystanders and drones.

(3) Airspace. Stakeholders relevant to the airspace must be informed as necessary. P4 says: "As you know, helicopters, they can enter and they do fly through uncontrolled airspace as well, which is generally where I fly. So, it's good practice to look at planned flights in your area as well as to report what you're going to do in that area to other airmen." This may involve reviewing information sources, and contacting stakeholders. P9 exemplifies: "[We may need] permission of any land owners... We've had to put in our own NOTAM (a notice to airmen), let other aircraft know that there's a UAV operating in the area." This might mean that pilots have to file a lot of paperwork or consents from different authorities, as P6 recounts: "The film commission, the City and County of San Francisco, the police department, the fire department, the Port Authority, the Coast Guard, Parks... Like, all of those authorities have to sign off on our stuff. So it's about creating a document that everyone looks at and goes 'Okay. These guys know what they're doing. I'm okay with it."'

(4) Equipment, Procedures and Parameters. Pilots decide on their equipment, procedures, and parameters. What route should the drone follow to take the pictures and videos? Several planned the route and overall mission to allow batteries to be switched at appropriate times, making sure to not run out as battery time is affected by wind etc. P10 always made sure that 25 percent of the battery would be left, after autonomous routes. What speed, height, direction, camera angle should the drone keep during the mission for the best shot? Where are the possible takeoff and landing points with good signal coverage? Often, a constellation of software and data answers these questions, including 3D models [P8], blueprints 


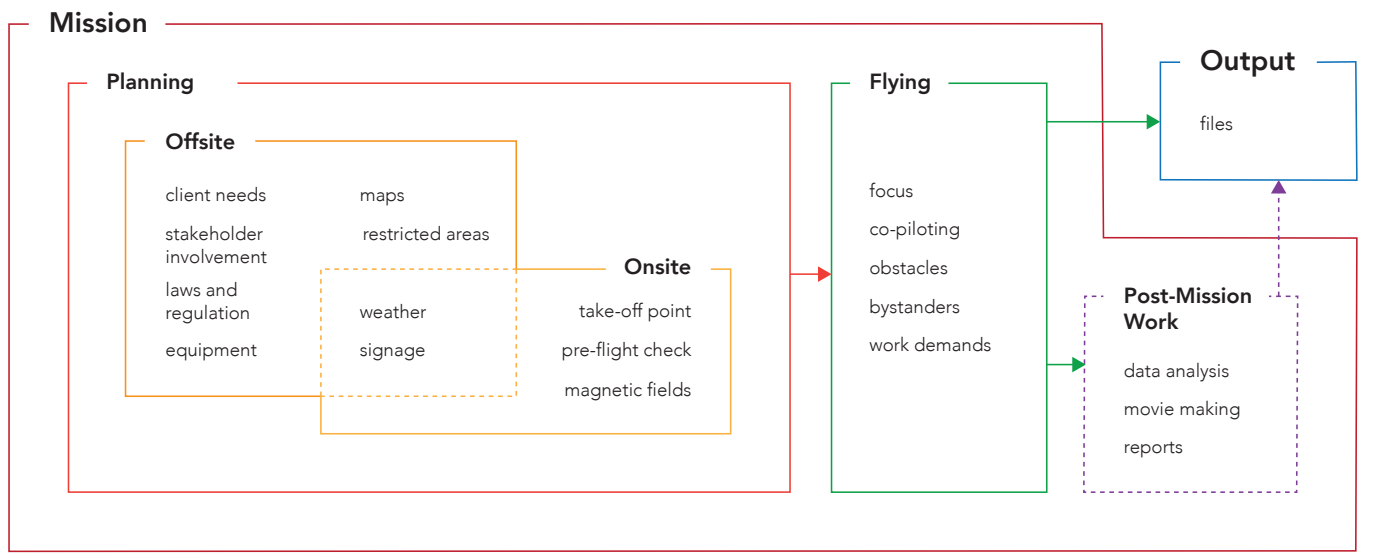

Figure 2: Overview of a typical drone mission. Planning, data, and business tasks seem to comprise most of the work for drone pilots.

[P10], and/or Google Earth [P6]. Pilots choose a specific drone from their fleet, depending on an interplay of factors like flight conditions, altitude, size, weight, payload, cost, and risks. Pilots - like P3, who only owns a smaller drone - might have to rent specific equipment at times, e.g. when a bigger drone is required to carry a specific payload. At times, they might also need to build or customize their equipment, which P8 illustrates: "We use some drones that are specialized for internal inspections in vessels. So that is, drones [with a] 360 [degree] cage around it. And we also have a bigger inspection drone that we have parachutes on, with the different cameras connected to it."

(5) Weather. Pilots always check forecasts before a mission, and continue watching the weather during flight. Even if some had experience of flying between rain showers when the weather was unstable, rain (or snow) and strong winds are hazardous and were avoided by all pilots.

(6) Privacy. Privacy was not an issue that figured into planning or was raised as an issue by the pilots. Still, they were aware that privacy could potentially be an issue. $\mathrm{P} 9$ recounted from an experience in real estate photography: "You're filming in a completely a different perspective and they see it in the air. They think you are spying on them." Some expressed that they made special configurations to avoid problems if potential bystanders would appear and have considerations about the mission. When privacy does figure in planning, it often has a regulatory or legal basis, or grounding in their prior experience. P9's policy when flying in urban settings is to "certainly, as a policy, contact the police beforehand, and inform the police of the operation. So if they get a call, they already know." In Europe, regulations also come into play. In P3's experience, "It's with the GDPR, so you have to inform then if it is the case that people happen to end up in the picture, for example, if I am scanning a building. Then you must also inform about it before, so those who pass the area have the opportunity to take another road or avoid entering the zone where there is a risk that they come in the picture." Design recommendations for HDI research: Develop an inclusive infrastructure for pilots. The findings suggests the necessity of developing an inclusive infrastructure that can scaffold a range of preparatory tasks by extracting, integrating, synthesizing and presenting critical information from various sources for targeted drone missions. The infrastructure should also provide decision support regarding route-planning and preliminary risk assessment in pursuit of a wider margin of safety.

4.2.2 On-site Preparations. Despite careful planning, many factors may require pilots to adapt to a current situation to reduce the risk to the drones and people. For example, regardless of the weather forecast, weather conditions must be kept under watch. Other possible disruptions must also be checked on-site, e.g. obstacles and magnetic influence nearby. Electronic interference may influence the compass and other instruments on the drone, decreasing the precision of flight controls. P8 describes how "before [they] do the flight, [they] have to look for transformators, radars, antennas and other equipment that has strong magnetic fields. [They] have to make sure there is no offshore cranes that are doing lifting in the area that we have a drone."

Several pilots would inform other stakeholders (including bystanders) on-site, before and during flight. They used various means, including yelling, signage, and notes placed on their vehicles. (This corroborates a research direction proposed in recent work, to investigate environmental signage in drone aviation [4].)

So once we have done pre-flight checks, we will pull the drone in position, get it ready to take off. Then we announce very loudly, very conspicuously, that the Drone is getting ready to go. Typically that's our [visual observer] yelling to everyone "drone is going up!" and you get everyone clear. [P6]

We will use, you know, the yellow clothes that shows that we are construction workers, and really try to show that we are holding the controls of the drones. So everyone that can see the drone will have the possibility to see the pilot too. And at some places when we've been out, construction sites would put up signs saying that there 
is drone work in progress, and whatever, and my contact information. [P10]

Pre-flight checks, sometimes with written checklist, are performed to ensure all components of the drone are in good condition. These involve going through "everything on the floor, visual inspection... checking telemetry and connections between the RC controller and actual drone" [P8]. P7 and colleagues "check the mechanical parts to ensure the connection is normal, check if bearing, transmission shaft, and motors make abnormal sound, if the propeller surface is clean and smooth, if any screws are loose, if the landing gear is normal..."

Design recommendation for HDI research: Support onsite preparations that include final checks, communication, signage and signaling to improve safety. The findings suggest that risk assessment is a priority and expertise dimension for pilots, which should be an indispensable part of the design of the drones and their underlying infrastructure. While very few research publications report how risk assessment, safety, liability protection, and similar concerns are implemented in HDI experiments [4], this study reveals that risk assessment is a main constituent of professional drone piloting work, forming as a critical basis for design. The practitioners wish to - and are expected to - foresee, recognize, and avert hazards that may damage people, the drone, or other assets, on site. An inclusive infrastructure that can scaffold a range of preparatory tasks by extracting, integrating, synthesizing and presenting critical information from various sources for targeted drone missions could be helpful. There is a need for flexibility and resilience to even unanticipated hazards and risks (such as weather). Moreover, communication must remain ongoing during drone missions, with complementing signage on operators and their team, on the drone, and in the setting.

\subsection{In Flight: Safety and Hazard Response}

Safety came up frequently in all interviews. Safety is the primary concern - pre-flight (per the above account of planning and preparation) and during flight. Here, we present the main issues and considerations that came up regarding safety.

4.3.1 Signal Integrity and Batteries. Signal loss or distortion (between drone and control) is a common technical issue that had jeopardized several drone operations, and was raising safety concerns among the pilots. The signal may break down completely, or disturbances can affect precision and stability of flight controls. This can result in a dangerous and stressful situation, as $\mathrm{P} 4$ pointed out: "We were basically flying next to a highway in Los Angeles, close to a highway but not over it, where there was a little hill that was between me and the drone. And I lost contact... Lost complete contact over a Los Angeles highway with a slightly larger drone than I use. I started freaking out." Recording a "home point" for the drone to return upon catastrophic signal loss was a common precaution for several pilots, which saved P4 in this situation. Similarly, knowing that batteries were about to run out could also result in a stressful situation. P9 described that: "if you're flying in the higher wind, that's obviously drain through your battery quicker". Design recommendation for HDI research: Support pilots to estimate the risk of signal loss and battery drain for missions in different types of conditions.
4.3.2 Focus and Division of Labor. Even if drones to some extent can fly almost entirely autonomously, this was typically not the situation for the professional pilots in the study. Some autonomous features were used occasionally by most; only P1 did several missions entirely autonomously. However, all missions still demanded attention. Thus, focus is an important quality to cultivate and prepare for, also when using autonomous features. P6 compares it to martial arts:

$$
\begin{aligned}
& \text { It's been, kind of, compared to martial artists (..) When } \\
& \text { you're in the air, you have to be that focused, and that } \\
& \text { intense, to maintain whatever it is that you're doing (...) } \\
& \text { So I try to create an environment where we are hyper } \\
& \text { focused on what we're doing, so we're safe and we're } \\
& \text { professional, and then once we land we can all kind of } \\
& \text { go back to our normal selves. } \\
& \text { [When flying autonomously,] "I can stop and I can talk } \\
& \text { to people. I don't have to worry about it." That's a really } \\
& \text { bad habit to have for a lot of new pilots... The Drone } \\
& \text { helps, assisting in flying, makes him feel like a better pi- } \\
& \text { lot than they are. And therefore they take a lot of things } \\
& \text { for granted. And for us, with higher stakes, we pay a } \\
& \text { lot of money for insurance, that kind of thing. We know, } \\
& \text { every second we're in the air we're held accountable. } \\
& \text { So we don't take anything lightly. Therefore it's really } \\
& \text { important that we set up cones (...) We let people know } \\
& \text { that, "look, this is a serious operation. fust hang in there } \\
& \text { with us. We're happy to talk with you. But right now } \\
& \text { we're very focused." }
\end{aligned}
$$

Focus can often be interrupted by bystanders who approach pilots because they are curious or interested. Engaging with people while piloting should be avoided at all times. Multiple participants noted that they always fly as a team of two or more, where one colleague takes on the duty of interacting with people who approach. According to $\mathrm{P} 3$ : "Most people are extremely curious, so it is completely hopeless to work at an event where there are a lot of people, because people are constantly coming forward and want to talk. Eh, that's why I never work alone at such events, so we are always two, because the one who flies just flies and has full focus on the drone and the other receives everyone who wants to ask a thousand questions." The duties of these team members are in fact various, including looking at checklists and documents, observing environments, operating certain components on the drone, and watching the equipment:

Typically, for us, it's pilot, sensor operator, and visual observer. When the drone's in the air the pilot and the sensor operator are focused in on the app. The visual observer is supposed to keep his eye on the machine, but they're also aware of our environment, watching the gear, that kind of thing. And so that person is also kind of in charge of crowd control. [P6]

We might have somebody operating the radio, while somebody else is speaking to the control tower, while somebody else is flying the drone. Or if we're flying where you're getting to the limit of your vision, when we're doing the agricultural job, for instance, I could still just see the drone, but you're flying to the end of 
the paddock, and you are getting near trees. Then we position an observer down that end, and you will be contacted by two-way radio, to make sure that your depth perception of where the drone is in relation to any obstacles, you know diminishes as you go further. [P9]

A notable exception was P4's approach in his artistic work, which diverges from the other practices: "I personally love shooting alone. If I'm really focused. Just like any other job, other people around, you get distracted. But I also love showing, having people look over my shoulder, explaining them the technology how it works, is also I think something I like to do. Kids love it. Places where I've been to on vacation where I fly, getting exotic, you know, views like in Spain or Costa Rica. I've had people just, like, flock around me. So the first few minutes I had like a couple people around, maybe like a family and a kids in Costa Rica. I remember 10 minutes later, there were 30 people around me because I could see just incredible stuff."

Design recommendation for HDI research: Consider that drone piloting conducted without a partner or a team can be difficult and unsafe, for example due to bystanders. Professionals' concerns and practices around how to maintain focus, show the necessity to distribute cognition and divide work in a team, to manage piloting and achieve needed communication with bystanders etc. This should inform future work, also for autonomous drones. Communicating drone state and intentions to bystanders is a research interest in HDI $[18,45]$. Future work may investigate and pick up inspirations from existing practices around how drone-flying teams prefer to operate.

4.3.3 Noise. Noise from a drone does not appear to hinder professional work. P9 stated: "it's obviously, I would say generally, not an issue." P5 had "not noticed that it has affected [them] in any way negatively." P8 noted that many of their work environments were already noisy, and drone noise does add to it very much. Unexpectedly, some pilots even brought up cases where noise was a positive factor, understood as a reduced risk. P1, working in populated industrial areas in China, said that noise can be helpful. "One reason is that the noise will keep the workers in the construction site alert. Another reason is that the management would want the workers to know that their work performance is being monitored." However, P10, working at construction sites in Norway, was instead concerned that noise could distract workers at construction sites, and lead to dangerous situations occurring in their normal job. For the police [P5], noise facilitates certain missions: "when we chase, for example... looking for people who may have run out into the terrain and stay away, that then they... they feel observed, regardless of whether we see them or not, so they think that we are up there. It can have an effect that we want."

From this perspective, noise is not necessarily a problem. However, pilots agreed that the noise can become an issue for bystanders or wildlife. P4 had this first hand: "I was flying outside, or on the border of the national forest, and a very sporty environmentalist woman came over and said, 'this is so annoying. The noise is bad. It's actually bad for the birds.' So she seemed to hate it as well, as she cared about the environment, so she didn't want me to fly there." On the other hand, noise generated by drones is a significant issue for many HDI research prototypes [4], but the exact interpretations and attitudes of stakeholders to noise varies between applications. In recent HDI research, drone sound has been reported as dimension of the user experience [27]. Design recommendation for HDI research: Drone noise concerns safety issues, power relations, and other interests among stakeholders and bystanders. The findings above indicate that sound as a phenomenon goes beyond user perceptions, revealing power relations and safety considerations of drone use.

4.3.4 Work Demands. Safety is the primary concern for pilots. But at the same time meeting business needs and delivering images at the necessary quality is also important. This clearly suggests another extra layer of complexity in a professional drone's work practice. Clear communication with the stakeholders is essential. Some pilots also have an arsenal of creative tactics developed with experience, to satisfy client demands while being cautious:

What people want and what you can do as an operator are two totally different things. So we have to explain to the people and say, "look, you know, I want to be safe. I want to get what you want." At the same time, you're not going to risk too much for the sake of your shot. So we just try to maintain a good level of professionalism, and try to keep the client happy. [P6]

I was in a school where they wanted to get the kids all lined up, to make a 60 by all lining up together, make 6-0 on the ground, and they wanted a photo of that. So we obviously couldn't get right over the top of them, but moving back and using a lens to make it look as though you were closer... I guess you just got to be a little bit conscious of other techniques, that you can make it look as though you're close, but not be as close. [P9]

There are jobs which demand high-risk maneuvers for the drone. This means pushing the limit of the balance between safety and needs. Some examples are found in cinematography: dynamic camera movements with a heavy drone, carrying expensive equipment exemplified by [P2] and [P6], and flying manually at high speed to film car races, exemplified by [P2]. Such jobs may require investing in niche skills and equipment. $\mathrm{P} 2$ had acquired and now explored flying with a custom racing drone to get longer shots of speeding cars. Overall, diverse concerns and stakeholders are involved in the work of pilots, posing challenges on their work demands. Design recommendation for HDI research: Consider proactive design and communication strategies for hazard prevention in high risk maneuvers. Drone failure can be costly both in terms of the equipment, but also the safety of users and bystanders. Therefore, it is of the utmost importance to consider a wide pool of possible hazards in missions and support pilots to communicate those to stakeholders and find alternative solutions to avoid high risks.

\subsection{Post-mission: Data Handling}

Handling data from a mission - processing, analysis, and reporting - extends the scope of pilots' work. Some pilots [e.g. P1, P8, P10] work within organizations with specific processes to handle the data. Some [e.g. P2, P3] often handle the entire workflow, up until the final deliverable. The involvement of pilots post-mission can 
be hands-on, as the job calls for their domain expertise (e.g. P10 as a geologist). Some might hand over the data to another team altogether (e.g. P6, as a director of photography who passes the data, in a particular format, to the editor). There are also missions with almost no data handling, only real-time monitoring and basic imaging. Police work is an example, comprising search missions or very basic photography that does not require post-processing [P5]. And though they acknowledge domain-specific expertise (over tactical piloting) as the value driver, pilots have varying degrees of interest in post-mission work. For P9, it is not a priority: “ $A$ lot of my stuff I guess is being at the front end of flying part and not so much of the production end... So I have done that side, but the majority of what I do is is really the capture, and then pass on the data, the raw data."

Data handling is often not routine. Stakeholder needs and pilots' involvement in data handling may vary from mission to mission. Often pilots are adept in using a range of software and other tools that add value to the job:

I use, eh... all programs are from, eh, Adobe. So I use Adobe Premiere Pro, called the movie editor... I edit the film and, and images, $u h$ in the editor... and send over to the customer then as they want it. [P2]

It depends a bit on the customer. Sometimes they want to process data themselves, then just hand over the material itself, whether it is film or photo, or a combination of film or photo. Then you hand over these files just to the client, then they process, because they can sometimes have systems like that I do not have access to. And they do not want anyone to process that. But then it could also be that I make an ortho-photo, so paste all these photos together into such a large map. And I do that in a software program, a GIS program then. If it's a movie, it can happen that I edit the movie. If someone, uh, a landowner wants to see his final felling, I then cut the film together in such cases and then send to the landowner. So it can be very different. There is nothing standardized, it is different. And when it comes to research, it is sometimes the case that in Excel you then produce different things and make tables and diagrams or something clear. [P3]

Challenges exist in handling of drone data: e.g. finding or developing specific software to perform certain tasks, tedious manual tasks which could potentially be automated, estimating and communicating the costs of data handling, and coordinating with different stakeholders. While current HDI research focuses on the flight itself, topics in post-flight work may be opportunities where future HDI research may create value. Pilots' statements below exemplify.

We have been in contact with both [Research Institutes] in Linköping and also Stockholm University. There are some, eh, guys and girls who research about that kind of thing, so hopefully, so if a person I want to collaborate with appears, who adds an AI software then, to interpret field material better or easier. So that you get away from the time-consuming manual review. [P3]

Often the clients will think, "after the drone mission everything should be in the report." But the drone gathers so much quality data, many gigabytes. So we need more time to look over the data. Sometimes it is important to get hold of the correct people from the client side you need data from coastal officers, telecom engineers, electrical engineers... [P8]

Overall, the forestry pilot, [P3], suggested that AI could add value to the tedious task of manually finding infected trees, a task part of the data handling. Meanwhile, the energy engineer, [P8] described how several stakeholders may be engaged in the data handling process. This suggests a need for HDI research on how to support data handling. Design recommendation for HDI research: The need for data handling depends on the type of mission and the industry and several stakeholders may be involved where support tools, such as AI, could reduce workload.

\subsection{Experience, Learning, and Extracurriculars}

The frequency of our pilots' drone missions varied between once every two weeks and more than seven missions per week. For many, piloting is only one among many professional responsibilities. For example, P10 had duties as a geologist and inspector at an engineering consultancy, the drone being but one tool that he utilizes in his job. Even those with piloting as their main responsibility have significant peripheral workloads, e.g. client relations and business development. The frequency of their drone operations also varied over time. P8, who typically works on inspecting oil and gas industry assets as well as bridges, sometimes flies 2-3 times per week, but also takes on periods of office work: "When working with offshore operations, then we are flying everyday. Then when we are coming back for maintenance, fault reporting something like that... It is more of a concentrating period flying drones."

Pilots agree that investing in training, certification, and continuous learning is worthwhile. The scope of their learning is broad, ranging across piloting techniques, peripheral software and systems, data analysis, and regulations. The core doctrine in training is that drones, albeit small, are aircraft; subject to many of the same considerations that apply to larger passenger- or cargo-bearing aircraft with whom they share the airspace. P9 gave the example of how he had to "be able to operate air band radio and talk to either other aircraft in a general capacity, or talk to the control tower controlled aerodrome." However, there is diversity in terms of the forms and systematicity of pilots' learning, depending on local regulations, their organization, and their industry. P5 explained that the Swedish police have developed their own curriculum, taught over ten days to cover "flight training with air regulations, air traffic radio, aviation system, flying procedures, flight training, principles, methodology. Yes, manual and night flying." P9 took part in a training program on his own accord, where "over five days you learn how to read aeronautical maps, you learn about the law, you learn about the operating principles of the aircraft, learn about the batteries. So there's a wide range of things that you that your cover, not just the ten dot points that you might get when you're on your own by a drone, and you know, don't fly closer than 30 meters from people and don't fly here." US-based participants had studied for and taken exams, as $\mathrm{P} 4$ recounts:

There are federal aviation laws that are under the jurisdiction of the FAA - Federal Aviation Administration. 
Table 3: Experience, Certification, and Equipment of our Participants

\begin{tabular}{lllll}
\hline & $\begin{array}{l}\text { Missions per Week } \\
\text { (approx.) }\end{array}$ & Years of Experience & Certificate & Equipment \\
\hline P1 & 1 & 3.5 & Licence from AOPA (China) & DJI (Phantom) \\
P2 & 3 & 2.5 & 1 year Commercial Pilot education (Sweden) & DJI (Inspire, Mavic) \\
P3 & 7 & 4 & 1 year Commercial Pilot education (Sweden) & DJI (Matrice, Phantom) \\
P4 & 3 & 5 & Drone pilot license, FAA (USA) & DJI (Inspire, Matrice, Mavic) \\
P5 & $1 / 2$ & 2 & Police-licensed UAS pilot (Sweden) & DJI (Matrice, Mavic) \\
P6 & $7+$ & 5 & Certified Commercial Drone Pilot (Part 107) (USA) & DJI (Inspire, Matrice, Phantom), xFold (Cinema, Dragon), Custom \\
P7 & $4+$ & 8 & License from AOPA (China) & DJI (Inspire, Phantom, S900) \\
P8 & 3 & 8 & Certified Operator & DJI (Matrice), Flyability (Elios) \\
P9 & 1 & 4 & RePL (Australia) & DJI (Matrice, Mavic, Phantom) \\
P10 & $1 / 2$ & 5 & Unofficial 2-day training & DJI (Matrice, Mavic, Phantom) \\
\hline
\end{tabular}

Things like, where you can fly, military operation zones, air spaces, airports... That dictates generally how you operate, at what elevation, what to pay attention to, across the federal airspace, and most of these rules are also global. For example drones globally are not allowed to fly over 400 feet. That's about a hundred and thirty meters. This is in the US as well as it's a global rule, but then federally the US has its own rules and operations. And then the Part 107 is the exam that allows... So the FAA administers many piloting exams and the ones that is for a drone pilots who want to run commercial operations is the Part 107. So there's multiple topics that I studied for that are covered under this under the Part 107 that I took.

Even as autonomous flight technologies proliferate, professionals believe that education and training is essential "the main way to avoid [accidents] is to train. Most of these are mistakes by less experienced people. Another way is to make the drones autonomous... But still the precondition is that the pilot is well trained, even with autonomous features" [P1].

For continuous learning and development, many participants habitually consult various online resources (apps, forums, YouTube, etc.), as well as more experienced peers. P6 also stressed the value of learning from the experts, reaching out to advanced drone pilots and manufacturers as a community: “.. It's really just going out and flying, and meeting people who are in the same circle."

In organizations where drone work is becoming increasingly in use (e.g. P10's structural engineering consultancy), internal networks are being built as support platforms. In P10's case this was a self-organized network of more than 10 pilots, distributed across Sweden, keeping contact via internal company communications. In another point, some pilots have strong opinions about how training and certification should be mandatory for all drone activities, including hobbyists and researchers. The primary concern is safety, but the reputation of their profession is seen to be at stake.
[People who lack training] can, like, lose control with the hand control, and they can run away and, and many may not really understand that a drone can actually be a potential death machine. If it falls down and ends up in the head, or in an inappropriate place, it's really dangerous. I do not think people are aware of it, therefore I believe that everyone who is going to fly drones must go some form of training. Hobby pilots should not have the same rights to fly at all, I was about to say. I do not think you should be able to fly drones without some form of training, which you can do today... I do not like hobby aviators because they destroy for us working professionally with drones. It is a giant scourge for us. And that, we are seldom taken seriously, we who work professionally with this, because the hobby pilots have, as it were, ruined the market a bit, which is sad. [P3]

A lot of hobby type of people may not want to pay that money and do a five day course to learn all the all the rules... And the hobby drone can give the commercial sector, I guess a bad look... If there's a couple of people not doing the right thing, then it gives a poor image, and that's a hard thing to overcome. [P9]

I am really looking forward to when we will have a complete system with the certification, or kind of driver's license, and identification system. That will be good improvement for everything. [P10]

In addition, a deeper issue in the lagging regulation was spotted; that it did not support the police's work: "It must be easier for the good in society to remove the bad machines in the air...get the legislation to keep up with...right now it is limited by the police's work for personal integrity... we do not do that today, because we have neither the technology nor the legal support for it" [P5].

Many professional pilots also take up flying drones as a leisure activity. Some (e.g. P2, P4, P8) have started out as hobbyists, and later began using drones professionally; and some (e.g. P10) took up 
drones as a professional tool, and later explored leisurely uses. P4, who shoots artistic photography, straddles the boundary, labeling himself a "prosumer." P2 mentioned a mistake he did as a beginner:

I would film horses too. It was not good. That, I had to cancel it immediately. It did not work. It was just personal, uh, fun, but it absolutely did not work. [laughs] They were terrified, those horses! [P2]

P10 would use a personal drone during leisure time, and then diverge from the best practices to experiment with new boundaries:

For example, when we were in the forests, we could use it daily... [We] marked each other and then try to run away from the drone or something. Try to have the Drone in front of you, and see how well it clears off trees... We use it quite a lot, all these different methods... To have a drone quite close to you instead of someone hanging around with a camera... So it's more of a toy and in that respect. Something to play with. I think it's a good way for [my daughter] to learn how to make movies. [P10]

Drones are a relatively new technology. It appears that the recent history of commercial applications is closely tied to the drone manufacturer DJI. While all of our participants used some kind of DJI drones, some also mentioned other vendors they used and developed for specific operations (indoor inspections and heavy payloads). The experiences of our participants thus related to the history of DJI's products. DJI's Phantom series of drones first launched in 2013, the Inspire series in 2014, the industrial Matrice series in 2015 , and the Mavic series in $2016 ;^{3}$ and the drone-flying experiences of our pilots range from 1-8 years (see table 3). Pilot training and on boarding are gaps in HDI research [4]. Interestingly, designing drone behaviors to communicate intuitively with naive users and bystanders [18, 29] are a design challenge that figure prominently in research. Future work might explore how to facilitate pilot training, designing tools and programs for learning best practices, and embedding best practices into interaction designs for drone products. Design recommendation for HDI research: Differentiating between hobbyist and professional piloting is needed, as well as regulations, training and networks to make any type of drone practice in society more safe.

\subsection{Expectations}

Our interviews with the pilots have also revealed expectations regarding technology, training, regulation, and market. Examples include:

- Public acceptance (e.g. P9) and market acceptance (e.g. P1)

- Regulations that restrict hobbyists (e.g. P3), keep up with the technological development (e.g. P6), and support professional practices (e.g. P5)

- Education and training support (e.g. P4)

- Software to automate data consumption, analysis, and interpretation (e.g. P3)

- Reliable and validated procedures for particular data collection and analysis scenarios (e.g. P6)

\footnotetext{
${ }^{3}$ https://www.djzphoto.com/blog/dji-product-history-timeline-drones-cameras-
} gimbals - accessed 2020-09-15.
- Incremental improvements over a constellation of related technologies: battery life, wireless communication, sensor resolution, mechanical fault diagnosis and tolerance, computer vision, etc.

Thus, while the HDI community tends to focus on moments where drone is in the air, our participants have diverse needs that figure before and after missions, as well as during. We see numerous opportunities for $\mathrm{HCI}$ research to create value at the intersections of traditional industries, emerging drone technologies, and hobbyist practices. Many of these opportunities are predicated on following the conventional HCI research wisdom of adopting user- and taskcentered approaches.

\section{DISCUSSION}

It is evident from the findings that the pilots' work is much more complex and requires knowledge and skills beyond manually controlling a drone. We observe that being a drone pilot is not an occupation in itself, but pilots are often experts within specific industries and knowledge domains, using drones as tools for delivering different kinds of data to meet specific needs. Safety and security are foremost in their drone missions, but they also have to meet specific needs of clients. In order to deliver drone missions in a safe and professional way, they use various resources to check pertinent regulations and inform relevant stakeholders that include authorities, nearby facilities, workers in the same organization, lay bystanders, clients, and more. The pilots were concerned about safety even from the planning and preparing stage. On site they had to take both internal factors (e.g. technological limitations) and external factors (e.g. new bystanders, weather, or even magnetic field effects) into account to assess the risks dynamically. These professionals are competent to satisfy all of these aspects primarily because they have had the training and education, so that they are proficient in drone operations and knowledgeable about the up-to-date regulations. However, we observed that their practice can also be challenged by public (e.g. privacy) and legal aspects (e.g. regulations that are lagging behind). Though every single use case is unique and may derive its own context-specific implications, the commonality emerged from the data unfolds an unexplored picture of the professional pilots' work - what actually matters to them and where such complexity resides.

This leads to the following design recommendations for HDI research:

- Carefully consider existing values, such as imaging, in industry to develop functionalities and applications of drones.

- Consider that professional pilots are foremost experts of a specific business and its values - piloting is second.

- Develop an inclusive infrastructure for pilots.

- Support on-site preparations: final checks, communication, signage and signaling to improve safety.

- Support pilots to estimate the risk of signal loss and battery drain for missions in different types of conditions

- Consider that drone piloting conducted without a partner or a team can be difficult and unsafe, for example due to bystanders.

- Drone noise concerns safety issues, power relations, and other interests among stakeholders and bystanders. 
- Consider proactive design and communication strategies for hazard prevention in high-risk maneuvers.

- The need for data handling depends on the type of mission and the industry and several stakeholders may be involved where support tools, such as AI, could reduce workload.

- Differentiating between hobbyist and professional piloting is needed, as well as regulations, training and networks to make any type of drone practice in society more safe.

The design recommendations, based on an empirical study of professional drone pilots, set an important empirical foundation for future development of professional practice, applications, training, regulations and legislation, and many other aspects in HDI research. Another future research area is to take a closer look at the interfaces used for piloting drones, from tablet-based displays to physical controllers. Moreover, a promising area for future research is to compare perspectives of professional and hobbyist practices to get a more holistic view of societal concerns and opportunities for HDI.

Our study has also explored how regulations are working out in practice and why informing diverse stakeholders is important, which is valuable to ground further regulatory development and necessary ethical considerations. Since the risk assessment (e.g. avoid flying close to people, animals and obstacles) and work tasks (e.g. use drones to conduct a surveillance mission by police) are supposed to be supported by regulations, it will create much value by delving into how an intended application may be affected by current laws and regulations. This also suggests that more researchers in $\mathrm{HCI}$ could engage in safety concerns when developing applications. More particularly, we find that highlighting a variety of situations of use and how a drone might be a safety risk can be part of research activities, such as actively engaging in potential use situations where children, cars, cyclists or other bystanders appear unexpectedly.

\subsection{Limitations}

Even if our drone pilots represent a wide range of industries, there are still many other industries that were not covered, such as delivery services and medical emergencies. Even if we found that our 10 participants successfully gave saturated findings and revealed shared concerns and practices, complementary studies are needed to reveal greater variations and details of practices within each industry. This is one limitation of the presented study, and future research could look more closely at each industry in order to get a more detailed and rich account of a specific practice.

Another limitation of our method is that the data is fully self reported, and insights only reveal what was considered to be significant or relevant by the participants themselves. We believe that the presented work opens up for future ethnographic observations, which may provide a more direct understanding of the practice and also support taking a critical perspective of what is actually happening on site when drones are in use.

Finally, it is also apparent the skewness of genders in our participant group of professional pilots is a limitation which can be further researched. An attempt to identify the causes that lead to a lack of gender diversity in the discipline appears warranted.

\section{CONCLUSION}

We present results from an interview study with 10 professional drone pilots, aiming to contribute a perspective on real-world, practical, and professional considerations - the state of practice - relevant for human-drone interaction (HDI). From pilots' accounts of their practices and considerations, we extracted implications and design recommendations for HDI research. To our knowledge, this is the first research on professional practices of drone pilots which maintains a focus on real use situations in industry, while expressly aiming to contribute to HCI and HDI research.

Highlights from these implications and our design recommendations are as follows:

(1) Safety is the top priority of professional drone pilots. In contrast, safety appears to be at best a secondary concern in HDI research, especially in empirical experiments. Future research can use the state of practice as a starting point for reinforcing this dimension.

(2) Professional drone applications are almost always predicated on industry-specific expertise; and the requirements of professional stakeholders differ from everyday use cases. HCI research has a rich tradition of ethnographic research, which may be applied across traditional industries to reveal novel value in $\mathrm{HDI}$.

(3) Only a small proportion of a professional pilot's work involves flying the drone. Preparations and industry specific tasks dominate. The concerns on safety also cover topics regarding training, regulatory development, norms at work etc. However, current HDI research almost exclusively focuses on flight moments. There are numerous opportunities for HDI research and design to expand beyond flight, taking a comprehensive and holistic system view of drone work.

The integration of these into future work can serve to reinforce the ecological validity of HDI research, align research concepts with current stakeholder requirements, create novel inventions, and expand the impact of research.

\section{ACKNOWLEDGMENTS}

We thank all participants in the study for their contribution to this research. We acknowledge the Wallenberg AI, Autonomous Systems and Software Program - Humanities and Society (WASPHS), funded by the Marianne and Marcus Wallenberg Foundation and the Marcus and Amalia Wallenberg Foundation. This research is funded by MediaFutures partners and the Research Council of Norway (grant number 309339).

\section{REFERENCES}

[1] Goodrich Michael A., Morse Bryan S., Gerhardt Damon, Cooper Joseph L., Quigley Morgan, Adams Julie A., and Humphrey Curtis. 2007. Supporting wilderness search and rescue using a camera-equipped mini UAV. fournal of Field Robotics 25, 1-2 (2007), 89-110. https://doi.org/10.1002/rob.20226

[2] Parastoo Abtahi, David Y. Zhao, Jane L. E., and James A. Landay. 2017. Drone Near Me: Exploring Touch-Based Human-Drone Interaction. Proc. ACM Interact. Mob. Wearable Ubiquitous Technol. 1, 3, Article 34 (Sept. 2017), 8 pages. https: //doi.org/10.1145/3130899

[3] Mauro Avila, Markus Funk, and Niels Henze. 2015. DroneNavigator: Using Drones for Navigating Visually Impaired Persons. In Proceedings of the 17th International ACM SIGACCESS Conference on Computers \&\#38; Accessibility (Lisbon, Portugal) (ASSETS '15). ACM, New York, NY, USA, 327-328. https://doi.org/10.1145/2700648.2811362 
[4] Mehmet Aydın Baytaş, Damla Çay, Yuchong Zhang, Mohammad Obaid, Asim Evren Yantaç, and Morten Fjeld. 2019. The Design of Social Drones: A Review of Studies on Autonomous Flyers in Inhabited Environments. In Proceedings of the 2019 CHI Conference on Human Factors in Computing Systems (Glasgow, Scotland Uk) (CHI '19). Association for Computing Machinery, New York, NY, USA, Article 250, 13 pages. https://doi.org/10.1145/3290605.3300480

[5] Mehmet Aydın Baytaş, Markus Funk, Sara Ljungblad, Jérémie Garcia, Joseph La Delfa, and Florian "Floyd" Mueller. 2020. IHDI 2020: Interdisciplinary Workshop on Human-Drone Interaction. In Extended Abstracts of the 2020 CHI Conference on Human Factors in Computing Systems Extended Abstracts (Honolulu, HI, USA) (CHI '20). Association for Computing Machinery, New York, NY, USA, 1-8. https://doi.org/10.1145/3334480.3375149

[6] Virginia Braun and Victoria Clarke. 2006. Using thematic analysis in psychology Qualitative research in psychology 3, 2 (2006), 77-101.

[7] Jones Brennan, Anthony Tang, and Carman Neustaedter. 2019. Drones for Remote Collaboration in Wilderness Search and Rescue. In 1st International Workshop on Human-Drone Interaction. Ecole Nationale de l'Aviation Civile [ENAC], Glasgow, United Kingdom. https://hal.archives-ouvertes.fr/hal-02128391

[8] Anke M. Brock, Jessica Cauchard, Markus Funk, Jérémie Garcia, Mohamed Khamis, and Matjaž Kljun. 2019. IHDI: International Workshop on Human-Drone Interaction. In Extended Abstracts of the 2019 CHI Conference on Human Factors in Computing Systems (Glasgow, Scotland Uk) (CHI EA '19). Association for Computing Machinery, New York, NY, USA, 1-7. https://doi.org/10.1145/3290607.3299001

[9] Anke M. Brock, Julia Chatain, Michelle Park, Tommy Fang, Martin Hachet, James A. Landay, and Jessica R. Cauchard. 2018. FlyMap: Interacting with Maps Projected from a Drone. In Proceedings of the 7th ACM International Symposium on Pervasive Displays (Munich, Germany) (PerDis '18). ACM, New York, NY, USA, Article 13, 9 pages. https://doi.org/10.1145/3205873.3205877

[10] Mira Crouch and Heather McKenzie. 2006. The logic of small samples in interview based qualitative research. Social Science Information 45, 4 (2006), 483-499. https://doi.org/10.1177/0539018406069584

[11] K. Dorling, J. Heinrichs, G. G. Messier, and S. Magierowski. 2017. Vehicle Routing Problems for Drone Delivery. IEEE Transactions on Systems, Man, and Cybernetics Systems 47, 1 (Jan 2017), 70-85. https://doi.org/10.1109/TSMC.2016.2582745

[12] Graham Dove, Kim Halskov, Jodi Forlizzi, and John Zimmerman. 2017. UX Design Innovation: Challenges for Working with Machine Learning as a Design Material In Proceedings of the 2017 CHI Conference on Human Factors in Computing Systems (Denver, Colorado, USA) (CHI '17). Association for Computing Machinery, New York, NY, USA, 278-288. https://doi.org/10.1145/3025453.3025739

[13] Federal Aviation Administration. 2020. Unmanned Aircraft Systems. In FAA Aerospace Forecast Fiscal Years 2020-2040. 41-63. https: //www.faa.gov/data_research/aviation/aerospace_forecasts/media/FY202040_FAA_Aerospace_Forecast.pdf. Accessed: 2020-08-30.

[14] Colin M. Gray. 2016. "It's More of a Mindset Than a Method": UX Practitioners' Conception of Design Methods. In Proceedings of the 2016 CHI Conference on Human Factors in Computing Systems (San Jose, California, USA) (CHI '16). Association for Computing Machinery, New York, NY, USA, 4044-4055. https://doi.org/10.1145/2858036.2858410

[15] Colin M. Gray, Erik Stolterman, and Martin A. Siegel. 2014. Reprioritizing the Relationship between HCI Research and Practice: Bubble-up and Trickle-down Effects. In Proceedings of the 2014 Conference on Designing Interactive Systems (Vancouver, BC, Canada) (DIS '14). Association for Computing Machinery, New York, NY, USA, 725-734. https://doi.org/10.1145/2598510.2598595

[16] Julia M Hildebrand. 2017. Situating hobby drone practices. Digital Culture \& Society 3, 2 (2017), 207-218.

[17] Javier Irizarry, Masoud Gheisari, and Bruce N Walker. 2012. Usability assessment of drone technology as safety inspection tools. Fournal of Information Technology in Construction (ITcon) 17, 12 (2012), 194-212.

[18] Walther Jensen, Simon Hansen, and Hendrik Knoche. 2018. Knowing You, Seeing Me: Investigating User Preferences in Drone-Human Acknowledgement. In Proceedings of the 2018 CHI Conference on Human Factors in Computing Systems (Montreal QC, Canada) (CHI '18). ACM, New York, NY, USA, Article 365, 12 pages. https://doi.org/10.1145/3173574.3173939

[19] Yunus Karaca, Mustafa Cicek, Ozgur Tatli, Aynur Sahin, Sinan Pasli, Muhammed Fatih Beser, and Suleyman Turedi. 2018. The potential use of unmanned aircraft systems (drones) in mountain search and rescue operations. The American fournal of Emergency Medicine 36, 4 (2018), 583 - 588 https://doi.org/10.1016/j.ajem.2017.09.025

[20] Kari Daniel Karjalainen, Anna Elisabeth Sofia Romell, Photchara Ratsamee, Asim Evren Yantac, Morten Fjeld, and Mohammad Obaid. 2017. Social Drone Companion for the Home Environment: A User-Centric Exploration. In Proceedings of the 5th International Conference on Human Agent Interaction (Bielefeld, Germany) (HAI '17). ACM, New York, NY, USA, 89-96. https://doi.org/10.1145/ 3125739.3125774

[21] Md. Nafiz Hasan Khan and Carman Neustaedter. 2019. An Exploratory Study of the Use of Drones for Assisting Firefighters During Emergency Situations. In Proceedings of the 2019 CHI Conference on Human Factors in Computing Systems (Glasgow, Scotland Uk) (CHI '19). Association for Computing Machinery, New
York, NY, USA, Article 272, 14 pages. https://doi.org/10.1145/3290605.3300502

[22] Md. Nafiz Hasan Khan and Carman Neustaedter. 2019. Exploring Drones to Assist Firefighters During Emergencies. In 1st International Workshop on HumanDrone Interaction. Ecole Nationale de l'Aviation Civile [ENAC], Glasgow, United Kingdom. https://hal.archives-ouvertes.fr/hal-02128386

[23] Ji-Eun Kim, Larry Kessler, Zach McCauley, Itsumi Niiyama, and Linda Ng Boyle. 2020. Human factors considerations in designing a personalized mobile dialysis device: An interview study. Applied Ergonomics 85 (2020), 103003. https://doi. org/10.1016/j.apergo.2019.103003

[24] Pascal Knierim, Steffen Maurer, Katrin Wolf, and Markus Funk. 2018. Quadcopterprojected in-situ navigation cues for improved location awareness. In Proceedings of the 2018 CHI Conference on Human Factors in Computing Systems. ACM, 433.

[25] Udo Kuckartz. 2014. Qualitative text analysis: A guide to methods, practice and using software. Sage.

[26] Udo Kuckartz and Stefan Rädiker. 2019. Analyzing qualitative data with MAXQDA. Springer.

[27] Joseph La Delfa, Mehmet Aydın Baytas, Rakesh Patibanda, Hazel Ngari, Rohit Ashok Khot, and Florian "Floyd" Mueller. 2020. Drone Chi: Somaesthetic Human-Drone Interaction. In Proceedings of the 2020 CHI Conference on Human Factors in Computing Systems (Honolulu, HI, USA) (CHI '20). Association for Computing Machinery, New York, NY, USA, 1-13. https://doi.org/10.1145/3313831. 3376786

[28] Florian 'Floyd' Mueller and Matthew Muirhead. 2015. Jogging with a Quadcopter. In Proceedings of the 33rd Annual ACM Conference on Human Factors in Computing Systems (Seoul, Republic of Korea) (CHI '15). ACM, New York, NY, USA, 2023-2032. https://doi.org/10.1145/2702123.2702472

[29] Mohammad Obaid, Felix Kistler, Gabrielè Kasparavičiūtė, Asim Evren Yantaç, and Morten Fjeld. 2016. How Would You Gesture Navigate a Drone?: A User-centered Approach to Control a Drone. In Proceedings of the 20th International Academic Mindtrek Conference (Tampere, Finland) (AcademicMindtrek '16). ACM, New York, NY, USA, 113-121. https://doi.org/10.1145/2994310.2994348

[30] Mohammad Obaid, Omar Mubin, Scott Andrew Brown, Asim Evren Yantac, Mai Otsuki, and Hideaki Kuzuoka. 2020. DroEye: Introducing a Social Eye Prototype for Drones. In Companion of the 2020 ACM/IEEE International Conference on Human-Robot Interaction (Cambridge, United Kingdom) (HRI '20). Association for Computing Machinery, New York, NY, USA, 378-380. https://doi.org/10.1145/ 3371382.3378313

[31] Lawrence A Palinkas, Sarah M Horwitz, Carla A Green, Jennifer P Wisdom, Naihua Duan, and Kimberly Hoagwood. 2015. Purposeful sampling for qualitative data collection and analysis in mixed method implementation research. Administration and policy in mental health and mental health services research 42 , 5 (2015), 533-544.

[32] Mirjana Prpa, Sarah Fdili-Alaoui, Thecla Schiphorst, and Philippe Pasquier. 2020. Articulating Experience: Reflections from Experts Applying MicroPhenomenology to Design Research in HCI. In Proceedings of the $2020 \mathrm{CHI}$ Conference on Human Factors in Computing Systems. 1-14.

[33] Agoston Restas. 2015. Drone applications for supporting disaster management. World fournal of Engineering and Technology 3, 03 (2015), 316.

[34] David J. Roedl and Erik Stolterman. 2013. Design Research at CHI and Its Applicability to Design Practice. In Proceedings of the SIGCHI Conference on Human Factors in Computing Systems (Paris, France) (CHI '13). Association for Computing Machinery, New York, NY, USA, 1951-1954. https://doi.org/10.1145/ 2470654.2466257

[35] Andrzej Romanowski, Sven Mayer, Lars Lischke, Krzysztof Grudzień, Tomasz Jaworski, Izabela Perenc, Przemyslaw Kucharski, Mohammad Obaid, Tomasz Kosizski, and Pawel W. Wozniak. 2017. Towards Supporting Remote Cheering During Running Races with Drone Technology. In Proceedings of the 2017 CHI Conference Extended Abstracts on Human Factors in Computing Systems (Denver, Colorado, USA) (CHI EA '17). ACM, New York, NY, USA, 2867-2874. https: //doi.org/10.1145/3027063.3053218

[36] Herbert J Rubin and Irene S Rubin. 2011. Qualitative interviewing: The art of hearing data. sage.

[37] Benjamin Saunders, Julius Sim, Tom Kingstone, Shula Baker, Jackie Waterfield, Bernadette Bartlam, Heather Burroughs, and Clare Jinks. 2018. Saturation in qualitative research: exploring its conceptualization and operationalization. Quality \& quantity 52, 4 (2018), 1893-1907.

[38] Jürgen Scheible and Markus Funk. 2016. In-situ-displaydrone: Facilitating Colocated Interactive Experiences via a Flying Screen. In Proceedings of the 5th ACM International Symposium on Pervasive Displays (Oulu, Finland) (PerDis '16). ACM, New York, NY, USA, 251-252. https://doi.org/10.1145/2914920.2940334

[39] N. Sharkey. 2009. Death strikes from the sky: the calculus of proportionality. IEEE Technology and Society Magazine 28, 1 (Spring 2009), 16-19. https://doi. org/10.1109/MTS.2009.931865

[40] Maximilian Speicher, Brian D Hall, and Michael Nebeling. 2019. What is mixed reality?. In Proceedings of the 2019 CHI Conference on Human Factors in Computing Systems. 1-15.

[41] J. M. Teixeira, R. Ferreira, M. Santos, and V. Teichrieb. 2014. Teleoperation Using Google Glass and AR, Drone for Structural Inspection. In 2014 XVI Symposium 
on Virtual and Augmented Reality. 28-36. https://doi.org/10.1109/SVR.2014.42

[42] D. Tezza and M. Andujar. 2019. The State-of-the-Art of Human-Drone Interaction A Survey. IEEE Access 7 (2019), 167438-167454.

[43] Dante Tezza, Derek Caprio, Denis Laesker, and Marvin Andujar. 2020. Let's Fly! An Analysis of Flying FPV Drones Through an Online Survey. In Proceedings of the Interdisciplinary Workshop on Human-Drone Interaction (iHDI 2020).

[44] Christopher James Vincent and Ann Blandford. 2017. How do health service professionals consider human factors when purchasing interactive medical devices? A qualitative interview study. Applied Ergonomics 59 (2017), 114 - 122. https://doi.org/10.1016/j.apergo.2016.08.025

[45] Michael Walker, Hooman Hedayati, Jennifer Lee, and Daniel Szafir. 2018. Communicating Robot Motion Intent with Augmented Reality. In Proceedings of the 2018 ACM/IEEE International Conference on Human-Robot Interaction (Chicago, IL, USA) (HRI '18). ACM, New York, NY, USA, 316-324. https://doi.org/10.1145/ 3171221.3171253

[46] Yang Wang, Huichuan Xia, Yaxing Yao, and Yun Huang. 2016. Flying eyes and hidden controllers: A qualitative study of people's privacy perceptions of civilian drones in the US. Proceedings on Privacy Enhancing Technologies 2016, 3 (2016), 172-190.

[47] Oian Yang Alex Scuito, John Zimmerman, Jodi Forlizzi, and Aaron Steinfeld. 2018 Investigating How Experienced UX Designers Effectively Work with Machine Learning. In Proceedings of the 2018 Designing Interactive Systems Conference (Hong Kong, China) (DIS '18). Association for Computing Machinery, New York, NY, USA, 585-596. https://doi.org/10.1145/3196709.3196730

[48] Yaxing Yao, Huichuan Xia, Yun Huang, and Yang Wang. 2017. Free to Fly in Public Spaces: Drone Controllers' Privacy Perceptions and Practices. In Proceedings of the 2017 CHI Conference on Human Factors in Computing Systems (Denver, Colorado, USA) (CHI '17). Association for Computing Machinery, New York, NY, USA, 6789-6793. https://doi.org/10.1145/3025453.3026049

[49] Yaxing Yao, Huichuan Xia, Yun Huang, and Yang Wang. 2017. Privacy Mechanisms for Drones: Perceptions of Drone Controllers and Bystanders. In Proceedings of the 2017 CHI Conference on Human Factors in Computing Systems (Denver, Colorado, USA) (CHI '17). Association for Computing Machinery, New York, NY, USA, 6777-6788. https://doi.org/10.1145/3025453.3025907 